Bull. Egypt. Soc. Physiol. Sci. Vol. (41) tissue (1), $41-55$

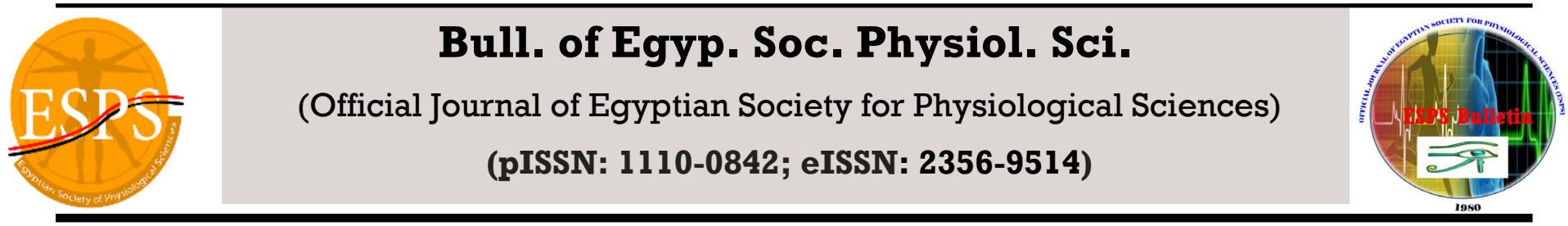

\title{
Effect of exercise and vitamin D on insulin like growth factor-1 level and skeletal muscle autophagy in diabetic rats
}

\author{
Sally M. Safwat ${ }^{1}$, Mohammed Ghalwash ${ }^{1}$, Nisreen M. Abo-EImaaty ${ }^{1}$, Emile F. Metias ${ }^{1}$, Basma \\ Osman $^{2}$ and Refka Messiha ${ }^{1}$ \\ ${ }^{1}$ Physiology department, faculty of medicine, Mansoura University, Mansoura, Egypt \\ ${ }^{2}$ Medical Experimental Research Center, Mansoura University, Mansoura, Egypt
}

Submit Date: April 12, 2020

Accept Date: May 1, 2020

Available Online: Jan 1, 2021

Keywords

- $\quad$ type II DM

- exercise

- $\quad$ vitamin D

- $\quad$ IGF-1

- $\quad$ LC3

\begin{abstract}
Background: diabetic myopathy is one of chronic complications of diabetes mellitus, which is evidenced by muscle weakness and atrophy. In our research we aimed to evaluate the protective role of exercise and vitamin D on diabetic induced changes in the skeletal muscles. Methods: Forty male Sprague Dawely rats were divided into control, diabetic, exercised diabetic, vitamin D treated diabetic and combined exercise and vitamin D diabetic rats. Fasting blood glucose, fasting insulin, HOMA-IR, serum IGF-1 level as well as the contractile properties of soleus and tibialis anterior muscles were measured. Also western blot analysis for measuring (LC3) and histopathological examination of both muscles were done. Results: in diabetic rats, fasting blood glucose, fasting insulin, HOMA-IR and LC3 (microtubule associated protein-1 light chain-3) expression in muscles were increased whereas serum IGF-1 level was decreased and the contractile properties of skeletal muscles were deteriorated. Vitamin D treatment decreased fasting blood glucose, fasting insulin, HOMA-IR and expression of LC3 but it increased serum IGF-1 with improvement in the skeletal muscles' contractility. Also exercised diabetic rats revealed improvement in all biochemical parameters, autophagy marker expression with further improvement in the skeletal muscles' contractility. Combining exercise and vitamin D treatment showed a more improvement in all studied parameters and the histopathological examination confirmed our results. Conclusion: regular exercise and vitamin D protect the muscles from diabetic induced lesions by improving glycemic state, serum level of IGF-1and suppressing autophagy which finally reflected on the contractile parameters of the muscles.
\end{abstract}

Corresponding author: Sally Mohamed Safwat, Faculty of medicine, Mansoura University, Mansoura, Egypt, E-mail: sallysafwat186@gmail.com, Phone: 01005515988 


\section{INTRODUCTION}

Diabetes mellitus (DM) is one of the public non-transmittable illnesses in the world and its occurrence is growing quickly worldwide [1]. It leads to many alternations in the skeletal muscles, as muscle weakness, fiber type transition and drop in energy metabolism [2]. Muscle atrophy is a recognizable problem of diabetes, frequently disturbs the lower limb muscles.

Autophagy is a process that includes cellular organelles turnover and destruction of cytoplasmic constituents during cellular stress [3]. It starts with formation of autophagosome that overwhelms cytoplasmic substances and attaches to lysosomes for destruction [4]. Materials incorporated within autophagolysosomes are needed to anabolic reactions to stabilize energy levels. Autophagy may lead to cell death when it is performed extremely during many pathological conditions [5]. It was reported that microtubule associated protein-1 light chain-3 (LC3) is involved in this process and its increase indicates increased autophagy. LC3 is found in many mammalian cells and it is produced into two forms; LC3-I and LC3II [6].

Insulin like growth factor-1 (IGF-1) is a hormone released by liver in reply to growth hormone [7]. It is a main controller of growth and metabolism. It is changed in many pathological conditions as DM [8]. Wang et al., [9] reported that diabetic patients have low level of IGF-1 and it is a therapeutic target.

Regular exercise is an effective treatment for the control of DM by enhancing metabolic status [10]. Though, the mechanisms underlying exercise induced adaptations in diabetic muscle still basically undefined.

Vitamin D is a fat-solute vitamin necessary for humans' growth and its non-classical actions are attracting more attention nowadays [11]. Accumulating evidence proposes that vitamin D owns anti-inflammatory, anti-oxidative, antihypertrophic and anti-fibrotic properties [12]. Furthermore, its deficiency has been linked to many musculoskeletal disorders like reduced muscle mass and diminished physical performance [13].

The aim of the current search is to assess the possible protective role of exercise and vitamin $\mathrm{D}$ on serum level of IGF-1, skeletal muscle autophagy and their subsequent impact on the skeletal muscles contractility in animal model of type II DM.

\section{Materials and methods}

\section{Experimental animals}

Fourty male Sprague dawely rats, aged 12-16 weeks, weighing nearly 200 gm were used. The animals were bought and lodged at Medical Experimental Research Centre (MERC), faculty of medicine, Mansoura University. Rats were accommodated in adequate comfortable environment. The research protocol was approved by the Mansoura Institutional Research Board (IRB), which follows the Declaration of Helsinki.

\section{Experimental design}

Animals were divided into 5 groups of 8 rats each. Group I (control): normal rats maintained on standard rat chow. Group II (DM): type II diabetic rats. Group III $(\mathrm{DM}+\mathrm{ex})$ : diabetic rats maintained on daily swimming exercise for 8 weeks [8]. Group IV (DM+vit D): diabetic rats maintained on vitamin D (10 IU/kg) by gavage for 8 weeks [14]. 
Group V (DM+ex+vit D): diabetic rats maintained on daily swimming exercise with vitamin $\mathrm{D}$ for 8 weeks.

Type II DM was induced by feeding rats high fat diet (60\% fat, $20 \%$ proteins, $20 \%$ carbohydrates) for 4 weeks then injected intraperitoneally by single low dose of streptozotocin $(35 \mathrm{mg} / \mathrm{kg}$ body weight) in $0.3 \mathrm{ml}$ citrate buffer solution [15].

\section{Swimming protocol}

Swimming exercise was conducted every day with no load in a water filled barrel and water temperature adjusted at $33-35^{\circ} \mathrm{C}$ [16]. Rats were acclimatized to swimming one week prior the experimental research to reduce animal stress in water [6] by starting the first swimming with duration limited to 15 minutes and increased daily till reaching 1h/day, 5 days/week for 8 weeks [8]. Following swimming rats were dried with towel and kept warm by heater.

\section{Tissue and blood sampling}

At the end of the experiment, overnight-fasted rats were anesthetized and blood was collected and centrifuged to obtain serum. Isolation of soleus and tibialis anterior muscles was done carefully. The muscles were immersed in Krebs solution in (mM) $120 \mathrm{NaCl} ; 25 \mathrm{NaHCO} 3: 1.2 \mathrm{NaH} 2 \mathrm{PO}$; 1.2 MgSO4; $5.0 \mathrm{KCI} ; 2.5$ calcium gluconate; 11.5 glucose at $30^{\circ} \mathrm{C}$, bubbled with a mixture of 5 $\% \mathrm{C} 02 ; 95 \% 02$, and $\mathrm{pH}$ 7.4. Muscles from one limb were used to record the contraction. Those of the other limb were frozen at $-80^{\circ} \mathrm{C}$ for western blot analysis.

\section{Muscle contractility recording}

The contractions of soleus and tibialis anterior muscles were recorded using the Biopac Student Lab 3.7.5 apparatus (MP36) data analysis unit (Inc., CA, USA) using supramaximal stimulation.
Single twitch $(1 \mathrm{~Hz})$ stimulus was used to detect the optimum length $\left(\mathrm{L}_{0}\right)$ of each muscle to be maintained for all subsequent stimulations. Separate single muscle twitches were recorded. For tetanus set the frequency to $40 \mathrm{~Hz}$, stimulate the tibialis anterior muscle for 1 second and the soleus muscle for 2 second. For fatigue determination stimulate the muscle at $40 \mathrm{~Hz}(1 \mathrm{sec}$ on, $1 \mathrm{sec}$ off for the tibialis anterior muscle and 2 sec on, $1 \mathrm{sec}$ off for the soleus muscle) until the force has decreased to $50 \%$ of the original [17]. The cross sectional area (CSA) of each muscle was detected by dividing the muscle's mass by the product of its optimum length and the density of mammalian muscle $(1.06 \mathrm{mg} / \mathrm{mm} 3)$ [18]. The specific tetanic tension was calculated by dividing tetanic tension by the calculated (CSA) [19].

\section{Biochemical analysis}

Plasma glucose was measured using kit purchased from (Crystal Chem company, USA). Plasma insulin was determined by utilizing rat insulin ELISA kit purchased from (Sun Red biological technology company, China) following the manufacturer's instructions. HOMA-IR (Homeostatic Model Assessment of Insulin Resistance) was calculated using the following formula: HOMA-IR $=$ glucose $(\mathrm{mmol} / \mathrm{L}) \times$ insulin $(\mu \mathrm{IU} / \mathrm{ml}) / 22.5$ [20]. Serum IGF-1 level was determined using rat ELISA kit purchased from (Chongqing biospes company, China) according to the manufacturer's instructions.

\section{Western blot analysis}

Western blot analysis was done using polycolonal anti- LC3 antibody kit purchased from Chongqing biospes company, China and was performed according to manufacturer's instructions [21]. 


\section{Histopathological examination}

Samples were taken from the muscles and fixed immediately in $10 \%$ formalin solution. Then samples were prepared to form paraffin sections to be stained with $H \& E$ according to Bancroft and Gamble [22] and examined by light microscopy.

\section{Statistical analysis}

The data was analyzed on SPSS version 17.0 (SAS 2004). The mean and standard error of mean (SE) values were calculated. Data between the groups were evaluated by using one way analysis of variance (ANOVA), followed by post-hoc Tukey HSD. $\mathrm{P}<0.05$ was considered statistically significant.

\section{Results}

\section{Biochemical results}

DM revealed significant increase in fasting blood glucose, fasting insulin and HOMA-IR as compared to control group $(\mathrm{p}<0.001)$. With vitamin $\mathrm{D}$ treatment rats exhibited significant decrease in these parameters in comparison with $\mathrm{DM}(\mathrm{p}<0.01)$. Also, in $\mathrm{DM}+\mathrm{ex}$ these parameters were significantly decreased as compared to DM, $\mathrm{DM}+$ vit $\mathrm{D} \quad(\mathrm{p}<0.001)$. In $\mathrm{DM}+\mathrm{ex}+\mathrm{vit} \mathrm{D}, \mathrm{a}$ significant decrease was detected compared to $\mathrm{DM}, \mathrm{DM}+$ vit $\mathrm{D}, \mathrm{DM}+\mathrm{ex}(\mathrm{p}<0.001)$. Regarding IGF-1 level, it was significantly reduced when compared with control group $(\mathrm{p}<0.001)$. Significant increase was detected in $\mathrm{DM}+$ vit $\mathrm{D}$ $(\mathrm{p}<0.01)$ in comparison with $\mathrm{DM}$ and in $\mathrm{DM}+\mathrm{ex}$ compared to DM, DM+vit D $\quad(\mathrm{p}<0.001)$. $\mathrm{DM}+\mathrm{ex}+$ vit $\mathrm{D}$ showed a more significant increase in IGF-1 value compared to DM+ex $(p<0.001)$ but still significantly lower than control group $(\mathrm{p}<$ 0.001) (table 1).

\section{Western blot assay results}

\section{Soleus muscle}

In DM LC3 I expression was significantly increased compared to control group $(\mathrm{p}<0.001)$. It was significantly decreased in $\mathrm{DM}+$ vit $\mathrm{D}$ when compared with DM $(p<0.001)$ and in DM+ex compared with DM, DM+vit D $(p<0.05)$. The best results were demonstrated in $\mathrm{DM}+\mathrm{ex}+\mathrm{vit} \mathrm{D}$ which showed significant reduction in LC3 I compared with other diabetic groups $(\mathrm{p}<0.001)$ but insignificant change with control group (table 2, figure 1a)

It was noticed that LC3 II expression was significantly increased in DM in comparison with control group $(\mathrm{p}<0.001)$. With vitamin $\mathrm{D}$ treatment it was significantly decreased when compared to DM ( $p<0.001)$. Also it was significantly decreased in $\mathrm{DM}+\mathrm{ex}$ as compared to $\mathrm{DM}, \mathrm{DM}+$ vit $\mathrm{D}(\mathrm{p}<0.05)$. The most obvious decrease in it was detected in $\mathrm{DM}+\mathrm{ex}+\mathrm{vit} \mathrm{D}$ compared to other diabetic groups $(p<0.001)$ but insignificant change with control group (table 2, figure 1a).

\section{Tibialis anterior muscle}

As compared to control group ( $\mathrm{p}<0.001)$, LC3 I expression in DM was significantly increased. It was significantly decreased in $\mathrm{DM}+$ vit $\mathrm{D}$ as compared to DM ( $\mathrm{p}<0.001)$. Further decrease was noticed in DM+ex when compared with DM, DM + vit $\mathrm{D}(\mathrm{p}<0.001)$. The most evident reduction was in $\mathrm{DM}+\mathrm{ex}+$ vit $\mathrm{D}$ compared to other diabetic groups $(\mathrm{p}<0.001)$ but still significantly higher than control group $(p<0.001)$ (table $2 \&$ figure 1b).

LC3 II expression was significantly increased in $\mathrm{DM}$ as compared to control $(\mathrm{p}<0.001)$. While it was decreased significantly in DM+vit D 
compared to DM $(\mathrm{p}<0.001)$ and in $\mathrm{DM}+\mathrm{ex}$ compared to DM, DM+vit D $(\mathrm{p}<0.05)$. Also it was significantly decreased in DM+ex+vit D when compared with DM, DM+vit D, DM+ex (p < $0.001)$ but insignificant change when compared to control group (table $2 \&$ figure $1 \mathrm{~b}$ ).

Table 1: Fasting blood glucose level, fasting insulin level, HOMA-IR and serum IGF-1

\begin{tabular}{cccccc} 
& $\begin{array}{c}\text { Control } \\
\mathbf{n = 8}\end{array}$ & $\begin{array}{c}\mathbf{D M} \\
\mathbf{n = 8}\end{array}$ & $\begin{array}{c}\mathbf{D M}+\mathbf{e x} \\
\mathbf{n = 8}\end{array}$ & $\begin{array}{c}\mathbf{D M}+\mathbf{8} i t \mathbf{D} \\
\mathbf{n}=\mathbf{8}\end{array}$ & $\begin{array}{c}\mathbf{D M}+\mathbf{e x}+\mathbf{v i t} \mathbf{D} \\
\mathbf{n}=\mathbf{8}\end{array}$ \\
\hline $\begin{array}{c}\text { Fasting blood } \\
\text { glucose }(\mathrm{mg} / \mathrm{dl})\end{array}$ & $87.87 \pm 5.74$ & $374.2 \pm 40.28^{\mathrm{a}}$ & $222 \pm 11.83^{\mathrm{a}, \mathrm{b}}$ & $327.1 \pm 35.23^{\mathrm{a}, \mathrm{b}, \mathrm{c}}$ & $182.4 \pm 12.24^{\mathrm{a}, \mathrm{b}, \mathrm{c}, \mathrm{d}}$ \\
\hline $\begin{array}{c}\text { Fasting insulin } \\
(\mu \mathrm{IU} / \mathrm{ml})\end{array}$ & $11.45 \pm 0.39$ & $23.49 \pm 1.51^{\mathrm{a}}$ & $15.81 \pm 0.91^{\mathrm{a}, \mathrm{b}}$ & $19.57 \pm 0.53^{\mathrm{a}, \mathrm{b}, \mathrm{c}}$ & $12.59 \pm 0.42^{\mathrm{b}, \mathrm{c}, \mathrm{d}}$ \\
\hline HOMA-IR & $1.47 \pm 0.05$ & $10.24 \pm 2.06^{\mathrm{a}}$ & $3.49 \pm 0.13^{\mathrm{a}, \mathrm{b}}$ & $6.12 \pm 1.09^{\mathrm{a}, \mathrm{b}, \mathrm{c}}$ & $1.6 \pm 0.07^{\mathrm{b}, \mathrm{c}, \mathrm{d}}$ \\
\hline $\begin{array}{c}\text { Serum IGF-1 } \\
(\mathrm{ng} / \mathrm{ml})\end{array}$ & $191.2 \pm 12.66$ & $92.48 \pm 3.17^{\mathrm{a}}$ & $128.9 \pm 3.07^{\mathrm{a}, \mathrm{b}}$ & $105.5 \pm 3.21^{\mathrm{a}, \mathrm{b}, \mathrm{c}}$ & $156.7 \pm 5.93^{\mathrm{a}, \mathrm{b}, \mathrm{c}, \mathrm{d}}$
\end{tabular}

Values are expressed as mean \pm SD. IGF-1: (Insulin like growth factor-1). DM+vit D: vitamin D treated diabetic group, DM+ex: exercised diabetic group, DM+ex+vit D: diabetic group with exercise and vitamin D treatment. (a): significance relative to control group, (b): significance relative to DM group, (c): significance relative to DM+ex group, (d): significance relative to $\mathrm{DM}+$ vit $\mathrm{D}$ group.

Table 2: the expression of $\mathrm{LC} 3$ I \& II relative to $\beta$-actin in the soleus and tibialis anterior muscles

\begin{tabular}{|c|c|c|c|c|c|}
\hline & $\begin{array}{c}\text { Control } \\
n=8\end{array}$ & $\begin{array}{l}\mathrm{DM} \\
\mathrm{n}=8\end{array}$ & $\begin{array}{c}\mathrm{DM}+\mathrm{ex} \\
\mathrm{n}=8\end{array}$ & $\begin{array}{c}\mathrm{DM}+\text { vit D } \\
\mathrm{n}=8\end{array}$ & $\begin{array}{c}\mathrm{DM}+\mathrm{ex}+\text { vit } \mathrm{D} \\
\mathrm{n}=8\end{array}$ \\
\hline LC3 I expression in soleus & $0.3 \pm 0.04$ & $4.66 \pm 0.69^{\mathrm{a}}$ & $2.36 \pm 0.27^{\mathrm{a}, \mathrm{b}}$ & $3.16 \pm 0.49^{\mathrm{a}, \mathrm{b}, \mathrm{c}}$ & $0.68 \pm 0.15^{\mathrm{b}, \mathrm{c}, \mathrm{d}}$ \\
\hline LC3 II expression in soleus & $0.36 \pm 0.06$ & $5.52 \pm 0.92^{\mathrm{a}}$ & $2.33 \pm 0.5^{\mathrm{a}, \mathrm{b}}$ & $3.22 \pm 0.29^{\mathrm{a}, \mathrm{b}, \mathrm{c}}$ & $0.66 \pm 0.03^{b, c, d}$ \\
\hline $\begin{array}{c}\text { LC3 I expression in tibialis } \\
\text { anterior }\end{array}$ & $0.68 \pm 0.06$ & $8.32 \pm 0.23^{\mathrm{a}}$ & $3.57 \pm 0.42^{\mathrm{a}, \mathrm{b}}$ & $4.46 \pm 0.43^{\mathrm{a}, \mathrm{b}, \mathrm{c}}$ & $2 \pm 0.45^{\mathrm{a}, \mathrm{b}, \mathrm{c}, \mathrm{d}}$ \\
\hline $\begin{array}{l}\text { LC3 II expression in tibialis } \\
\text { anterior }\end{array}$ & $0.59 \pm 0.05$ & $9.63 \pm 0.76^{\mathrm{a}}$ & $5.15 \pm 0.38^{\mathrm{a}, \mathrm{b}}$ & $6.3 \pm 0.76^{\mathrm{a}, \mathrm{b}, \mathrm{c}}$ & $1.32 \pm 0.63^{\mathrm{b}, \mathrm{c}, \mathrm{d}}$ \\
\hline
\end{tabular}

Values are expressed as mean \pm SD. LC3: (microtubule associated protein-1 light chain-3). DM: diabetic group, DM+vit D: vitamin D treated diabetic group, $\mathbf{D M}+\mathbf{e x}$ : exercised diabetic group, $\mathbf{D M}+\mathbf{e x}+\mathbf{v i t} \mathbf{D}$ : diabetic group with exercise and vitamin D treatment. (a): significance relative to control group, (b): significance relative to DM group, (c): significance relative to $\mathrm{DM}+$ ex group, (d): significance relative to $\mathrm{DM}+$ vit $\mathrm{D}$ group.

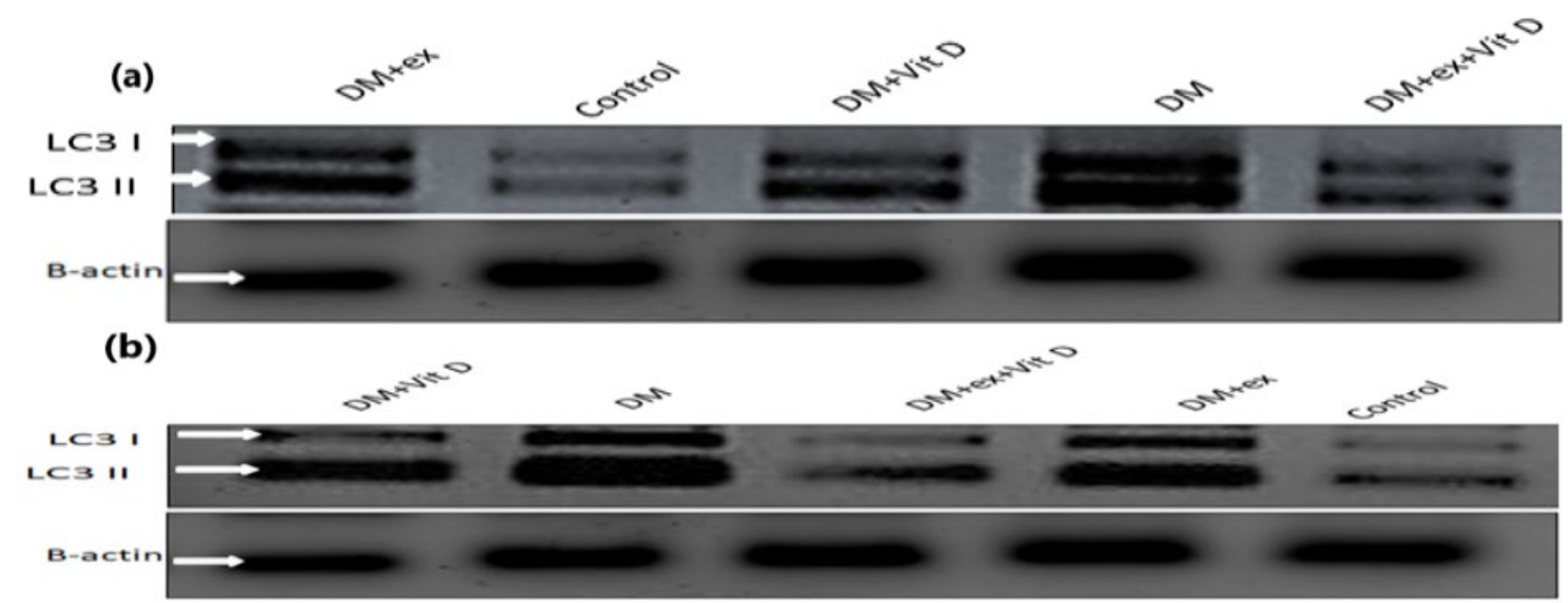

Figure 1: LC3 (microtubule associated protein-1 light chain-3) expression in (a) soleus muscle \& in (b) tibialis anterior muscle. DM: diabetic group, DM+vit D: vitamin D treated diabetic group, DM+ex: exercised diabetic group, DM+ex+vit $\mathrm{D}$ : diabetic group with exercise and vitamin $\mathrm{D}$ treatment. 
III. Skeletal muscle contractility results

\section{Soleus muscle}

Isometric twitch tension: it was significantly reduced in DM group when compared to control group ( $\mathrm{p}<0.001)$. This decline was significantly improved in $\mathrm{DM}+$ vit $\mathrm{D}$ in comparison with $\mathrm{DM}$ $(\mathrm{p}<0.05)$ and in $\mathrm{DM}+\mathrm{ex}$ compared with $\mathrm{DM}$, $\mathrm{DM}+$ vit $\mathrm{D}(\mathrm{p}<0.05)$. The best improvement was detected in $\mathrm{DM}+\mathrm{ex}+\mathrm{vit} \mathrm{D}$ in comparison with other diabetic groups $(\mathrm{p}<0.05)$. However it became insignificant when compared with control group (table 3, figure $2 \mathrm{~A}$ ).

\section{Time to peak twitch and half relaxation time:}

time to peak twitch in soleus muscle was significantly prolonged in DM compared to control group ( $\mathrm{p}<0.001)$. On the other hand either in $\mathrm{DM}+$ vit $\mathrm{D}$ or $\mathrm{DM}+\mathrm{ex}$ group, it showed significant decrease compared with DM $(p<0.001)$. Further improvement was noticed in $\mathrm{DM}+\mathrm{ex}+\mathrm{vit} \mathrm{D}$ compared with other diabetic groups $(\mathrm{p}<0.001)$ but insignificant change with control group. Also half relaxation time was significantly increased in DM compared to control group ( $\mathrm{p}<0.001$ ). It was significantly decreased in DM+vit D compared to $\mathrm{DM}(\mathrm{p}<0.001)$ and in $\mathrm{DM}+\mathrm{ex}$ compared to DM, $\mathrm{DM}+$ vit $\mathrm{D}(\mathrm{p}<0.01)$. $\mathrm{DM}+\mathrm{ex}+\mathrm{vit} \mathrm{D}$ showed the best significant reduction in its value as compared to other diabetic groups $(\mathrm{p}<0.05)$ but insignificant change with control group (table 3 ).

\section{Tetanic tension and specific tetanic tension:} there was significant decrease in tetanic tension in DM when compared to control $(\mathrm{p}<0.001)$. It was significantly increased in DM+vit D as compared to DM $(p<0.05)$. Also it was significantly increased in DM+ex compared to DM, DM+vit D $(\mathrm{p}<0.05) . \mathrm{DM}+\mathrm{ex}+\mathrm{vit} \mathrm{D}$ revealed a significant increase in it in comparison with DM, DM+vit D,
$\mathrm{DM}+\mathrm{ex}(\mathrm{p}<0.01)$, however a significant decrease was detected as compared to control group $(\mathrm{p}<0.05)$ (table 3 \& figure 2B). Regarding the specific tetanic tension, it also demonstrated a significant decrease in DM as compared with control group $(p<0.001)$. On the other hand it showed a significant increase in $\mathrm{DM}+$ vit $\mathrm{D}$ in comparison with DM $(\mathrm{p}<0.05)$. In $\mathrm{DM}+\mathrm{ex}$, it revealed a significant increase when compared to $\mathrm{DM}, \quad \mathrm{DM}+\mathrm{vit} \quad \mathrm{D} \quad(\mathrm{p}<0.05) . \quad \mathrm{DM}+\mathrm{ex}+\mathrm{vit} \quad \mathrm{D}$ demonstrated a more significant increase in specific tetanic tension as compared to DM, $\mathrm{DM}+$ vit $\mathrm{D}, \mathrm{DM}+\mathrm{ex}(\mathrm{p}<0.01)$. However non significant change with control group was detected (table 3).

Time to fatigue: it showed significant decrease in DM when compared to control group $(\mathrm{p}<0.001)$. However, it was significantly increased in $\mathrm{DM}+$ vit $\mathrm{D}$ in comparison with $\mathrm{DM}(\mathrm{p}<0.01)$. Further prolongation was observed in $\mathrm{DM}+\mathrm{ex}$ compared to DM, DM+vit D $(\mathrm{p}<0.05)$. $\mathrm{DM}+\mathrm{ex}+\mathrm{vit}$ $\mathrm{D}$ also showed further increase when compared with $\mathrm{DM}, \mathrm{DM}+$ vit $\mathrm{D}, \mathrm{DM}+\mathrm{ex}(\mathrm{p}<0.01)$ but it still significantly lower than control group $(\mathrm{p}<0.001)$ (table 3, figure 2 C).

\section{Tibialis anterior muscle}

Isometric twitch tension: it was significantly decreased in DM in comparison with control group $(\mathrm{p}<0.001)$. Its value was significantly increased in $\mathrm{DM}+$ vit $\mathrm{D}$ as compared to $\mathrm{DM}(\mathrm{p}<0.01)$ and in $\mathrm{DM}+\mathrm{ex}$ when compared to DM, DM+vit D $(\mathrm{p}<0.05)$. The most obvious increase was noticed in $\mathrm{DM}+\mathrm{ex}+\mathrm{vit} \mathrm{D}$ in comparison with $\mathrm{DM}, \mathrm{DM}+\mathrm{vit}$ $D$, DM +ex $(p<0.05)$, but showed significant decrease compared to control group $(\mathrm{p}<0.001)$ (table $4 \&$ figure $3 \mathrm{~A}$ ). 
Table (3): The contractile properties of soleus muscle

\begin{tabular}{|c|c|c|c|c|c|}
\hline & $\begin{array}{c}\text { Control } \\
\mathbf{n}=\mathbf{8}\end{array}$ & $\begin{array}{l}\text { DM } \\
\mathrm{n}=\mathbf{8}\end{array}$ & $\begin{array}{c}\mathbf{D M}+\mathbf{e x} \\
\mathbf{n}=\mathbf{8}\end{array}$ & $\begin{array}{c}\text { DM+vit D } \\
n=8\end{array}$ & $\begin{array}{c}\text { DM+ex+vit D } \\
n=8\end{array}$ \\
\hline $\begin{array}{l}\text { Isometric twitch } \\
\text { tension }(\mathrm{gm})\end{array}$ & $4.98 \pm 1.82$ & $0.25 \pm 0.06^{\mathrm{a}}$ & $2.89 \pm 0.46^{\mathrm{a}, \mathrm{b}}$ & $1.56 \pm 0.24^{\mathrm{a}, \mathrm{b}, \mathrm{c}}$ & $4.37 \pm 0.71^{\mathrm{b}, \mathrm{c}, \mathrm{d}}$ \\
\hline $\begin{array}{l}\text { Time to peak } \\
\text { twitch }(\mathrm{sec})\end{array}$ & $0.07 \pm 0.004$ & $1.14 \pm 0.22^{\mathrm{a}}$ & $0.35 \pm 0.05^{\mathrm{a}, \mathrm{b}}$ & $0.59 \pm 0.09^{\mathrm{a}, \mathrm{b}, \mathrm{c}}$ & $0.09 \pm 0.005^{b, c, d}$ \\
\hline $\begin{array}{l}\text { Half relax.time } \\
(\mathrm{sec})\end{array}$ & $0.09 \pm 0.007$ & $0.27 \pm 0.02^{\mathrm{a}}$ & $0.13 \pm 0.006^{\mathrm{a}, \mathrm{b}}$ & $0.16 \pm 0.005^{\mathrm{a}, \mathrm{b}, \mathrm{c}}$ & $0.11 \pm 0.01^{\mathrm{b}, \mathrm{c}, \mathrm{d}}$ \\
\hline $\begin{array}{l}\text { Tetanic tension } \\
(\mathrm{gm})\end{array}$ & $12.58 \pm 3.67$ & $0.63 \pm 0.19^{\mathrm{a}}$ & $6.19 \pm 0.81^{\mathrm{a}, \mathrm{b}}$ & $3.55 \pm 0.66^{\mathrm{a}, \mathrm{b}, \mathrm{c}}$ & $9.61 \pm 0.89^{a, b, c, d}$ \\
\hline $\begin{array}{c}\text { Specific tetanic } \\
\text { tension } \\
\left(\mathrm{gm} / \mathrm{mm}^{3}\right) \\
\end{array}$ & $0.86 \pm 0.26$ & $0.1 \pm 0.05^{\mathrm{a}}$ & $0.49 \pm 0.08^{\mathrm{a}, \mathrm{b}}$ & $0.29 \pm 0.06^{\mathrm{a}, \mathrm{b}, \mathrm{c}}$ & $0.74 \pm 0.09^{\mathrm{b}, \mathrm{c}, \mathrm{d}}$ \\
\hline $\begin{array}{l}\text { Time to fatigue } \\
(\mathrm{sec})\end{array}$ & $26.25 \pm 5.18$ & $5.25 \pm 0.46^{\mathrm{a}}$ & $14.25 \pm 1.04^{\mathrm{a}, \mathrm{b}}$ & $10.38 \pm 0.74^{\mathrm{a}, \mathrm{b}, \mathrm{c}}$ & $18.75 \pm 2.32^{\mathrm{a}, \mathrm{b}, \mathrm{c}, \mathrm{d}}$ \\
\hline
\end{tabular}

Values are expressed as mean \pm SD. DM: diabetic group, DM+vit D: vitamin D treated diabetic group, DM+ex: exercised diabetic group, $\mathbf{D M}+\mathbf{e x}+\mathbf{v i t} \mathbf{D}$ : diabetic group with exercise and vitamin D treatment. (a): significance relative to control group, (b): significance relative to DM group, (c): significance relative to DM+ex group, (d): significance relative to $\mathrm{DM}+$ vit D group.
A) Isometric twitch tension
B) Tetanic tension
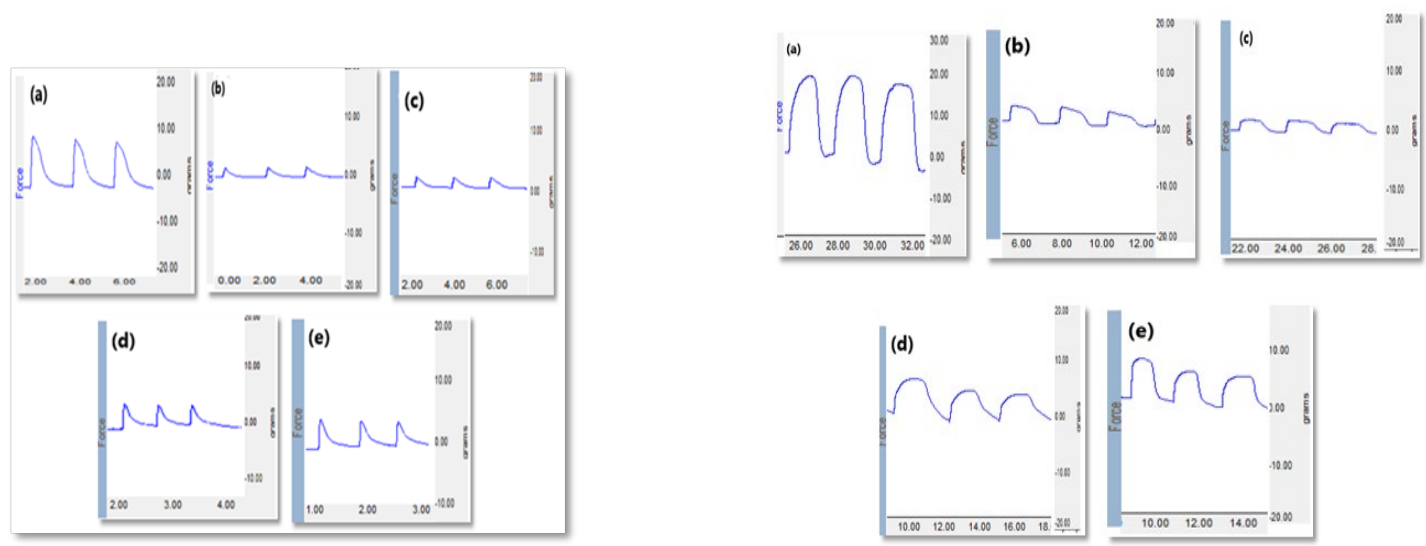

C) Time to fatigue
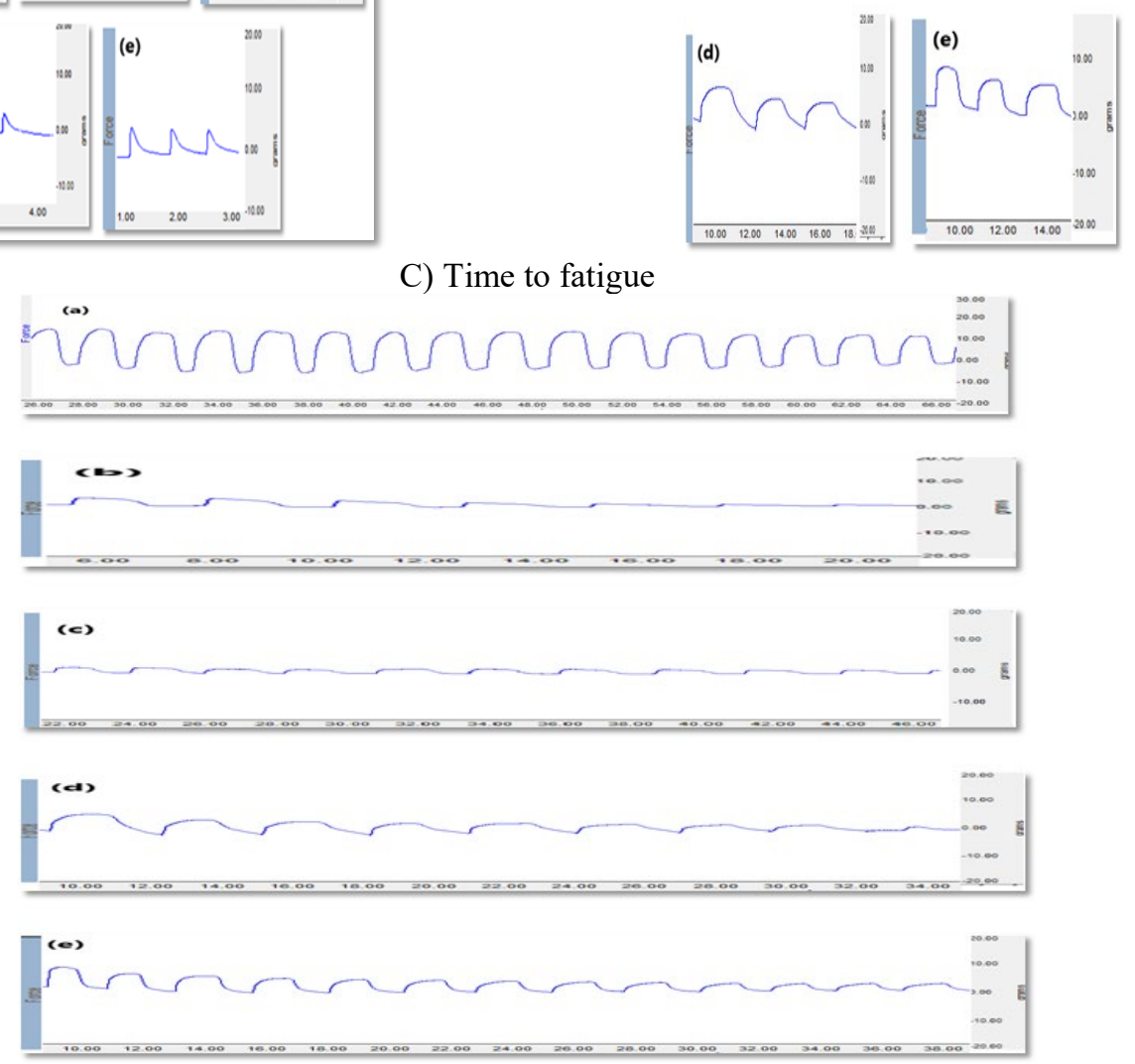

Figure 2: recording of contractile properties of soleus muscles. a) control group, (b) diabetic group, (c) vitamin D treated diabetic group (d) exercised diabetic group, (e): diabetic group with exercise and vitamin D treatment. 


\section{Time to peak twitch and half relaxation}

time: time taken by the muscle to reach peak twitch was significantly increased in DM as compared to control group ( $\mathrm{p}<0.001)$. In $\mathrm{DM}+\mathrm{vit}$ $D$, it was significantly decreased when compared with DM $(\mathrm{p}<0.01)$. DM+ex showed significant decrease in its value compared to DM, DM+vit D $(p<0.001)$. A significant reduction was also detected in $\mathrm{DM}+\mathrm{ex}+$ vit $\mathrm{D}$ in comparison with $\mathrm{DM}$, $\mathrm{DM}+$ vit $\mathrm{D}, \mathrm{DM}+\mathrm{ex}(\mathrm{p}<0.01)$, however nonsignificant change was observed when compared with control group (table 4). Regarding half relaxation time, it was significantly increased in DM compared to control group $(\mathrm{p}<0.001)$. $\mathrm{DM}+$ vit D significantly decreased it compared to DM ( $<<0.001)$. Daily swimming decreased it compared to DM, DM+vit D $(p<0.05)$. Further decrease was observed in $\mathrm{DM}+\mathrm{ex}+\mathrm{vit} \mathrm{D}$ when compared to other diabetic groups $(\mathrm{p}<0.001)$, but no significant change when compared to control group (table 4).

\section{Tetanic tension and specific tetanic tension:} tetanic tension was significantly decreased in DM in comparison with control group $(\mathrm{p}<0.001)$. It was significantly increased in DM+vit D compared to $\mathrm{DM}(\mathrm{p}<0.05)$. Further increase was detected in $\mathrm{DM}+\mathrm{ex}$ in comparison with $\mathrm{DM}, \mathrm{DM}+\mathrm{vit} \mathrm{D}$ $(p<0.05)$. Also there was a significant increase in it in $\mathrm{DM}+\mathrm{ex}+\mathrm{vit} \mathrm{D}$ when compared to other diabetic groups $(\mathrm{p}<0.01)$, but still significantly lower than control group $(p<0.001)$. In addition, specific tetanic tension was significantly decreased in DM compared to control group ( $\mathrm{p}<0.001)$. On the other hand, it was significantly increased in $\mathrm{DM}+$ vit $\mathrm{D}$ compared to $\mathrm{DM}(\mathrm{p}<0.001)$ and in $\mathrm{DM}+\mathrm{ex}$ in comparison with $\mathrm{DM}, \mathrm{DM}+\mathrm{vit} \mathrm{D}$ $(p<0.01) . \quad D M+e x+i t \quad D$ revealed the best improvement as compared to other diabetic groups ( $\mathrm{p}<0.001$ ), but insignificant change with control group (table 4, figure $3 \mathrm{~B}$ ).

Time to fatigue: as compared to control group, it was significantly decreased in DM $(p<0.001)$. This was increased significantly in $\mathrm{DM}+\mathrm{vit} \mathrm{D}$ in comparison with DM $(\mathrm{p}<0.001)$. Also in $\mathrm{DM}+\mathrm{ex}$, it was increased compared to DM, DM+vit D (p < 0.001). More significant increase was detected in $\mathrm{DM}+\mathrm{ex}+\mathrm{vit} \mathrm{D}$ as compared to other diabetic groups ( $\mathrm{p}<0.01$ ), but still significantly lower than control group $(\mathrm{p}<0.001)$ (table $4 \&$ figure $3 \mathrm{C})$.

\section{Histopathological examination}

Soleus muscle in DM group showed vacuolated fibers and widened interstitial tissue spaces (figure 4 b) compared to control. Improvement was detected in $\mathrm{DM}+$ vit $\mathrm{D}$ and muscle exhibited mild interstitial edema and mildly degenerated fibers (figure 4c). Exercised diabetic rats showed minor leukocytic infiltration, focal hyalinosis and hypertrophied nuclei (figure 4 d). Muscle in $\mathrm{DM}+\mathrm{ex}+\mathrm{vit} \mathrm{D}$ group appeared near normal in structure (figure $4 \mathrm{e}$ ).

In tibialis anterior muscle of DM group, there is disruption in its normal structure with appearance of markedly widened interstitial tissue and myophagia (figure $5 \mathrm{~b}$ ). DM+vit $\mathrm{D}$ exhibited excess pale eosinophilic fluid in interstitium with widened interstitial spaces (figure 5 c). Mild regeneration with accumulation of mild eosinophilic fluid in interstitium and hyalinized muscle fibers appeared in DM+ex (figure $5 \mathrm{~d}$ ). While in $\mathrm{DM}+\mathrm{ex}+\mathrm{vit} \mathrm{D}$ there is retaining of near normal muscle structure but with very mild interstitial edema (figure $5 \mathrm{e}$ ). 
Table 4: the contractile properties of tibialis anterior muscle

\begin{tabular}{cccccc} 
& $\begin{array}{c}\text { Control } \\
\mathbf{n = 8}\end{array}$ & $\begin{array}{c}\mathbf{D M} \\
\mathbf{n = 8}\end{array}$ & $\begin{array}{c}\mathbf{D M}+\mathbf{e x} \\
\mathbf{n = 8}\end{array}$ & $\begin{array}{c}\mathbf{D M}+\mathbf{8} \text { it } \mathbf{D} \\
\mathbf{n = 8}\end{array}$ & $\begin{array}{c}\mathbf{D M}+\mathbf{e x}+\mathbf{v i t} \mathbf{D} \\
\mathbf{n = 8}\end{array}$ \\
\hline $\begin{array}{c}\text { Isometric twitch } \\
\text { tension }(\mathrm{gm})\end{array}$ & $14.16 \pm 2.51$ & $2.31 \pm 0.54^{\mathrm{a}}$ & $7.37 \pm 0.95^{\mathrm{a}, \mathrm{b}}$ & $5.13 \pm 0.94^{\mathrm{a}, \mathrm{b}, \mathrm{c}}$ & $9.64 \pm 1.49^{\mathrm{a}, \mathrm{b}, \mathrm{c}, \mathrm{d}}$ \\
\hline $\begin{array}{c}\text { Time to peak } \\
\text { twitch (sec) }\end{array}$ & $0.02 \pm 0.001$ & $0.37 \pm 0.07^{\mathrm{a}}$ & $0.11 \pm 0.01^{\mathrm{a}, \mathrm{b}}$ & $0.19 \pm 0.03^{\mathrm{a}, \mathrm{b}, \mathrm{c}}$ & $0.02 \pm 0.002^{\mathrm{b}, \mathrm{c}, \mathrm{d}}$ \\
\hline $\begin{array}{c}\text { Half relax.time } \\
(\text { sec })\end{array}$ & $0.06 \pm 0.004$ & $0.17 \pm 0.02^{\mathrm{a}}$ & $0.08 \pm 0.004^{\mathrm{a}, \mathrm{b}}$ & $0.1 \pm 0.003^{\mathrm{a}, \mathrm{b}, \mathrm{c}}$ & $0.07 \pm 0.009^{\mathrm{b}, \mathrm{c}, \mathrm{d}}$ \\
\hline $\begin{array}{c}\text { Tetanic tension } \\
(\text { gm) }\end{array}$ & $28.57 \pm 6.54$ & $4.14 \pm 1.39^{\mathrm{a}}$ & $14.02 \pm 0.99^{\mathrm{a}, \mathrm{b}}$ & $9.35 \pm 0.83^{\mathrm{a}, \mathrm{b}, \mathrm{c}}$ & $19.65 \pm 1.72^{\mathrm{a}, \mathrm{b}, \mathrm{c}, \mathrm{d}}$ \\
\hline $\begin{array}{c}\text { Specific tetanic } \\
\text { tension } \\
(\text { gm } / \text { mm3) }\end{array}$ & $1.43 \pm 0.29$ & $0.29 \pm 0.08^{\mathrm{a}}$ & $0.91 \pm 0.06^{\mathrm{a}, \mathrm{b}}$ & $0.59 \pm 0.12^{\mathrm{a}, \mathrm{b}, \mathrm{c}}$ & $1.29 \pm 0.09^{\mathrm{b}, \mathrm{c}, \mathrm{d}}$ \\
\hline $\begin{array}{c}\text { Time to fatigue } \\
(\text { sec })\end{array}$ & $16.5 \pm 1.85$ & $3.63 \pm 0.52^{\mathrm{a}}$ & $9.13 \pm 0.64^{\mathrm{a}, \mathrm{b}}$ & $6.5 \pm 0.53^{\mathrm{a}, \mathrm{b}, \mathrm{c}}$ & $11 \pm 0.93^{\mathrm{a}, \mathrm{b}, \mathrm{c}, \mathrm{d}}$
\end{tabular}

A) Isometric twitch tension
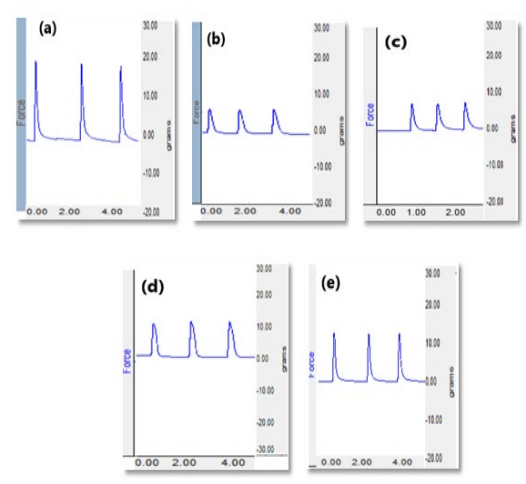

B) Tetanic tension
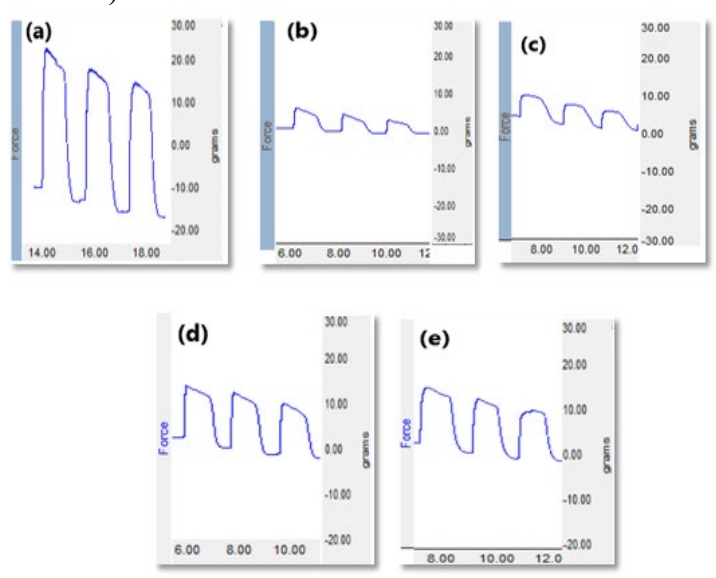

C) Time to fatigue
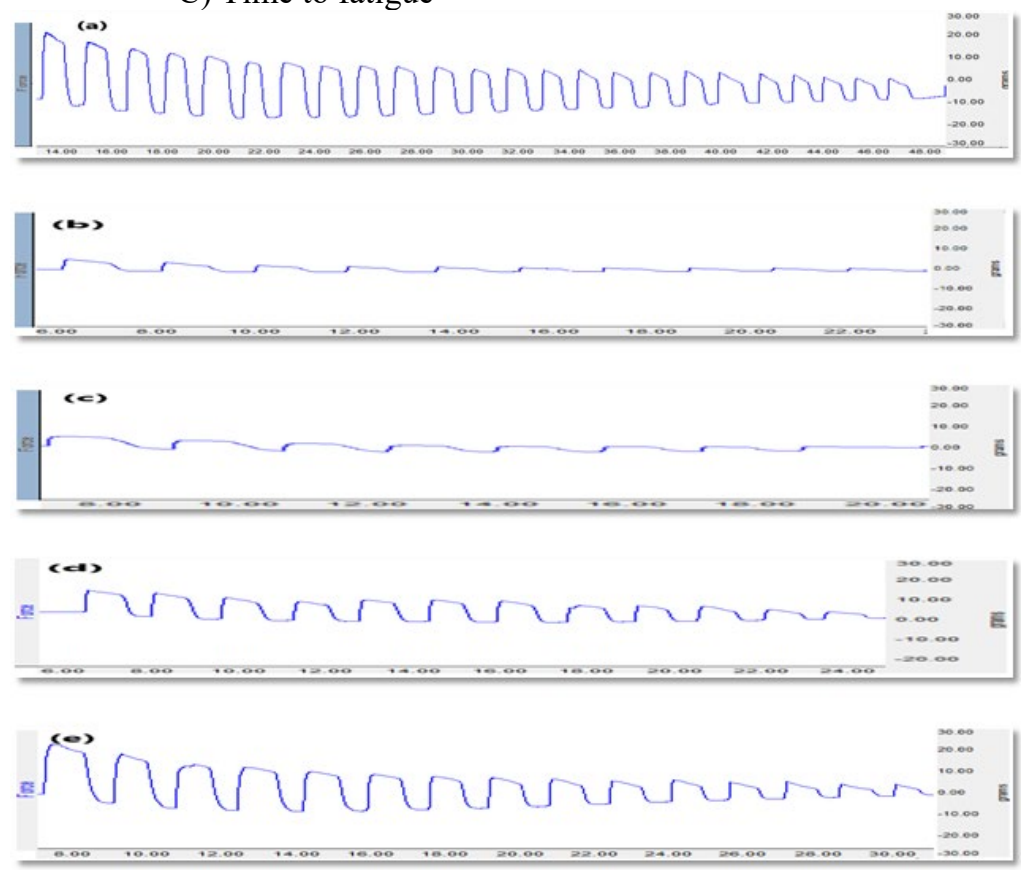

Figure 3: recording of contractile properties of tibialis anterior muscles. a) control group, (b) diabetic group, (c) vitamin $\mathrm{D}$ treated diabetic group $(\mathrm{d})$ exercised diabetic group, (e): diabetic group with exercise and vitamin D treatment. 


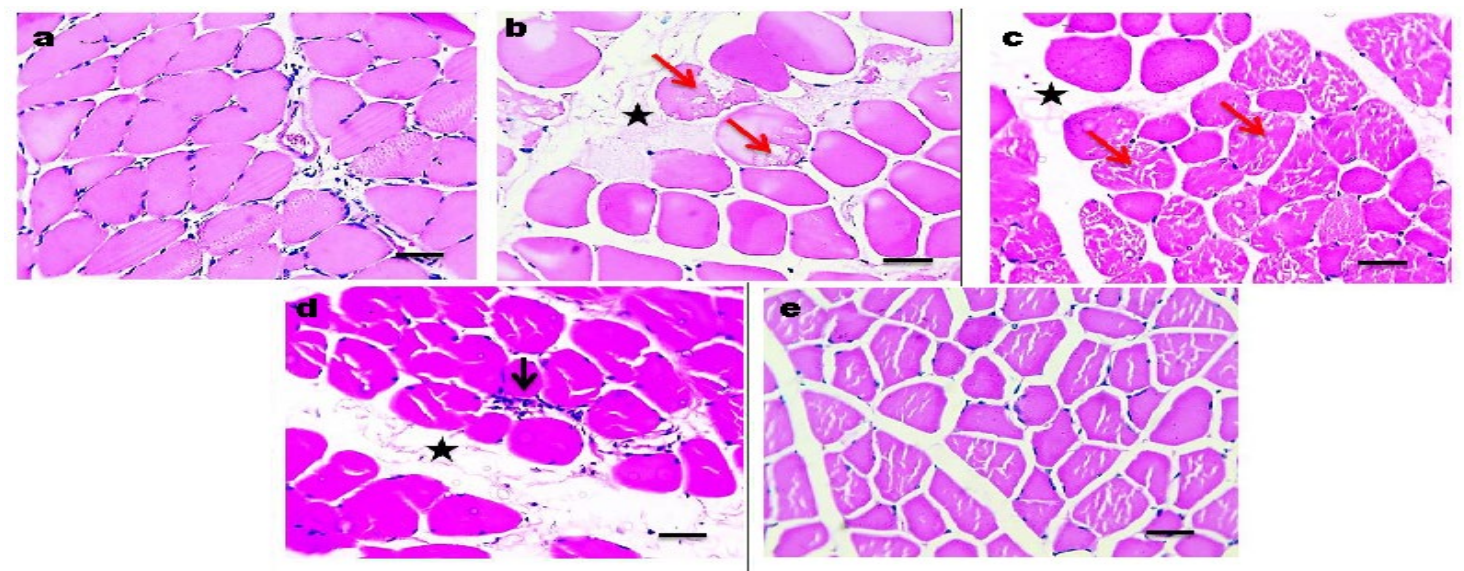

Figure 4: histopatholgical changes in soleus muscle. (a) Control group. (b) Diabetic group: red arrows: vacuolated fibers, asterisks: edema. (c) Vitamin D treated diabetic group: red arrows: degenerated fibers, asterisks: edema. (d) Exercised diabetic group: black arrow: leukocytic infiltration, asterisks: mild edema. (e) Diabetic group with exercise and vitamin D treatment: normal muscle picture. H\&E, X: 400 bar 50.

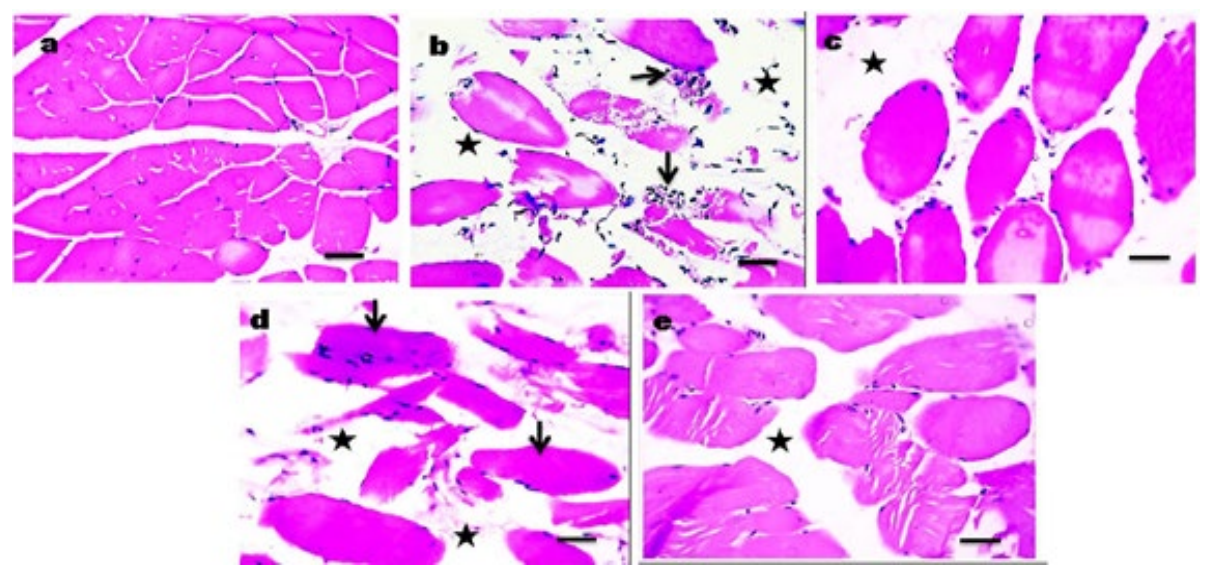

Figure 5: histopathological changes of tibialis anterior muscles. (a) Control group. (b) Diabetic group: asterisks: marked edema, arrows: myophagia. (c) Vitamin D treated diabetic group: asterisk: edema. (d) Exercised diabetic group: asterisk: edema, arrows: hyalinized fibers. (e) Diabetic group with exercise and vitamin D treatment: asterisks: very mild edema. H\&E, X: 400 bar 50.

\section{Discussion}

Diabetic myopathy is a clinical condition manifested by decline in the muscle mass, weakness, and general reduction in the physical capacity [23]. In our study blood glucose, plasma insulin and HOMA-IR were significantly increased in diabetic group as compared to control group and this was previously reported in many studies [24] [25]. Vitamin D has been reported to provide beneficial effects on insulin action by inducing the expression of insulin receptors and improving insulin responsiveness for glucose transport [26]. This could suggest the significant decline in blood glucose, plasma insulin and HOMA-IR in vitamin $\mathrm{D}$ treated rats; that was noticed in our study. Exercised diabetic rats also exhibited an improvement in the glycemic state compared to vitamin $\mathrm{D}$ treated rats. These results were in consistent with Mehta et al. [27] who stated that exercise plays an important role in the reduction of the high blood glucose by improving insulin action and enhancing blood glucose uptake independent on insulin. The combination of vitamin $\mathrm{D}$ and exercise in our study showed a synergistic action on blood glucose, plasma insulin and HOMA-IR 
and this suggests an additive effect of vitamin D to that of exercise on the control of these parameters. As regard serum level of IGF-1, diabetic rats showed significant decline in its level compared to control group. This may be related to the reduction in the hepatic responsiveness to growth hormone in DM with subsequent decrease in the expression of hepatic IGF-1mRNA [28]. He et al. [29] reported that vitamin D is highly likely to regulate IGF-1 concentrations by acting in the liver. This could explain our finding that vitamin D treated rats revealed significant elevation in their serum IGF-1 level compared to diabetic group. It has been shown in the present study that exercise also improves low levels of IGF-1 compared to vitamin $\mathrm{D}$ treated rats. This result was in agreement with Borges et al. [30] and may be due to increased IGF-1 mRNA synthesis. The combination of vitamin $\mathrm{D}$ treatment and exercise in diabetic rats provides more improvement in the level of IGF-1 and this may be due to their cooperative effects.

Regarding LC3 expression, it was noticed that the expression of LC3 I and LC3 II was significantly elevated in diabetic rats when compared to control group. It can be attributed to mTOR (mammalian target of rapamycin); a sensor of cell nutrient status; which is inhibited during stressful condition as in DM and its inhibition activates autophagy [31]. Their expression was significantly decreased in vitamin $\mathrm{D}$ treated rats in comparison with diabetic group. This result could be explained by the ability of vitamin D to regulate the expression of cyclin dependent kinase inhibitor in the cells which works as a cell growth regulator thus protecting them from autophagy induced death [32]. However, our results came in disagreement with $\mathrm{Wu}$ and Sun [33]. Exercised diabetic rats revealed a more significant reduction in LC3 expression when compared to vitamin D treated rats. This may be related to the beneficial effects of exercise in improving muscle metabolic state that could suppress diabetic skeletal muscle autophagy [6]. A more significant enhancement in LC3 I and LC3 II expression was observed in $\mathrm{DM}+\mathrm{ex}+\mathrm{vit} \mathrm{D}$ than other treated groups and this could be explained by the additive effect of exercise and vitamin $\mathrm{D}$ treatment.

It was obvious from our results that LC3 expression in the tibialis anterior muscle was higher than that in soleus muscle. The favored activation of autophagy in fast twitch muscle than slow twitch muscle is related mostly to the role of mTOR which is reported to be inhibited more in fast twitch than slow twitch muscle fibers [34]. Ogata et al. suggested that the difference in frequency of contraction between fast and slow muscle may contribute to mTOR regulation [34].

Regarding the contractile properties, the isometric twitch tension in soleus muscle of diabetic rats was significantly declined compared with control group and this was consistent with Virgen-Ortiz et al. [35]. Similar finding was recorded in the tibialis anterior muscle and this was previously observed by Huang et al. [36]. In diabetic skeletal muscles the sarcoplasmic reticulum (SR) and T tubules are disrupted and there is impairment of calcium release and in calcium sequestration [37]. This could explain our result which showed a significant increase in time taken to reach peak twitch and half relaxation time in soleus and tibialis anterior muscles in diabetic group compared with control group.

The maximum tetanic tension was significantly decreased in both muscles in diabetic rats in 
comparison with control group. This result could be explained by the low glycogen energy stores in diabetic muscles that leads to the reduction in amount of ATP needed for tetanic contraction [38]. The specific tetanic tension was detected to avoid the influence of muscle size growing larger with time. It was reduced significantly in diabetic group compared to control group and this could be explained by the fact that DM exhibited atrophy to both type of muscles [36].

Regarding time taken to fatigue, it was significantly shortened in diabetic group compared to normal rats. The increased fatigability in diabetic muscles related to the reduction in ATP and accumulation of metabolic end products that impair the functioning calcium handling apparatus [39].

It has been postulated that vitamin D improves calcium homeostasis in skeletal muscles, reduces inflammatory mediated myopathy and improves muscle contractile protein composition [40]. This could explain our finding which revealed that vitamin $\mathrm{D}$ treatment improved the contractile parameters of both muscles compared with diabetic rats.

In exercised diabetic rats, there was improvement in all studied contractile parameters of soleus and tibialis anterior muscles. Eshima et al. confirmed the same result and demonstrated that the muscular impairment in skeletal muscles mainly resulted from dysfunctional SR mechanisms and exercise training improves them [41]. Indeed, combining vitamin $\mathrm{D}$ and exercise revealed a more significant improvement in the contractility of both muscles due to their synergistic action.

As clearly apparent from our study, the contractile parameters of soleus and tibialis anterior muscles were significantly impaired in diabetic rats in comparison with control group and this was associated with higher expression of (LC3) and low levels of IGF-1. On the other hand, we noticed that the improvement in both muscles contractility was obtained with treatment of diabetic rats with vitamin $\mathrm{D}$ alone, exercise alone and more improvement in combination treatment with vitamin $\mathrm{D}$ and exercise. This enhancement in muscles contraction was also accompanied by reduction in autophagy expression in both muscles with elevation in serum level of IGF-1 and this may indicate the role of IGF-1 and autophagy in the maintenance the health state of the muscles.

Moreover, our results were confirmed by histopathological examination. In diabetic group there were many structural changes in both muscles like inflammatory infiltrate between muscle fibers, increased fibrosis and widened interstitial tissue spaces. These results were in agreement with Hany et al. [42]. Conversely there was improvement in these structural changes with vitamin D treatment. This may be due to its ability to enhance cell proliferation, increase muscle protein synthesis and to suppress apoptosis in the skeletal muscles [43]. Consistent improvement with exercise was also noticed. This may be due to the ability of exercise to adequately preserve the structures of myofibers owing to its anti-atrophic effects [6]. The muscles of diabetic rats receiving combined exercise and vitamin D treatment exhibited a more significant improvement in their structures and this indicates their importance.

\section{Conclusion}

It is possible to conclude that vitamin $\mathrm{D}$ and exercise has many beneficial effects on the skeletal muscles of diabetic rats. Their efficacy is mainly 
through increasing the low levels of IGF-1 and by suppressing the hyperactive autophagy noticed in the diabetic muscles. The combination of vitamin $\mathrm{D}$ and exercise has shown a more promising effect as they provide many synergistic effects.

Acknowledgements: Physiology Department and MERC are acknowledged for their significant contribution to the experimental part of the study.

Conflict of interest: The authors declare that there is no conflict of interest.

\section{References}

1. Hill $\mathbf{J}$ : Reducing the risk of complications associated with diabetes. Nursing Standard (through 2013) 23 (25): 49-55, 2009.

2. Fujimaki S, Wakabayashi T, Asashima M, Takemasa T, Kuwabara T: Treadmill running induces satellite cell activation in diabetic mice. Biochemistry and biophysics reports 8: 6-13, 2016.

3. Mizushima N: Autophagy: process and function. Genes \& development 21 (22): 2861-73, 2007.

4. Hayashi-Nishino M, Fujita N, Noda T, Yamaguchi A, Yoshimori T, Yamamoto A: A subdomain of the endoplasmic reticulum forms a cradle for autophagosome formation. Nature cell biology 11 (12): 1433-7, 2009.

5. Galluzzi L, Morselli E, Vicencio JM, Kepp O, Joza N, Tajeddine N, et al.: Life, death and burial: multifaceted impact of autophagy. Biochem Soc Trans 36: 786-90, 2008.

6. Lee $\mathbf{Y}$, Kim J-H, Hong $\mathbf{Y}$, Lee S-R, Chang K-T, Hong Y: Prophylactic effects of swimming exercise on autophagy-induced muscle atrophy in diabetic rats. Laboratory animal research 28 (3): 171-9, 2012.
7. Vahdatpour C, Dyer AH, Tropea D: Insulin-like growth factor 1 and related compounds in the treatment of childhood-onset neurodevelopmental disorders. Frontiers in neuroscience 10: 450-7, 2016.

8. Leme J, Silveira R, Gomes R, Moura R, Sibuya C, Mello M, et al.: Long-term physical training increases liver IGF-I in diabetic rats. Growth Hormone \& IGF Research 19 (3): 262-6, 2009.

9. Wang CY, Li XD, Hao ZH, Xu D: Insulin-like growth factor-1 improves diabetic cardiomyopathy through antioxidative and antiinflammatory processes along with modulation of Akt/GSK-3 $\beta$ signaling in rats. The Korean Journal of Physiology \& Pharmacology 20 (6): 613-9, 2016.

10. Teixeira de Lemos $\mathbf{E}$, Oliveira J, Páscoa Pinheiro J, Reis F: Regular physical exercise as a strategy to improve antioxidant and antiinflammatory status: benefits in type 2 diabetes mellitus. Oxidative medicine and cellular longevity 2012: 15, 2012.

11. Mitri J, Muraru M, Pittas A: Vitamin D and type 2 diabetes: a systematic review. European journal of clinical nutrition 65 (9): 1005-15, 2011.

12. Lee T-W, Lee T-I, Chang C-J, Lien G-S, Kao Y-H, Chao T-F, et al.: Potential of vitamin D in treating diabetic cardiomyopathy. Nutrition research 35 (4): 269-79, 2015.

13. Tieland M, Brouwer-Brolsma EM, Nienaber-Rousseau Cv, van Loon LJ, De Groot L: Low vitamin D status is associated with reduced muscle mass and impaired physical performance in frail elderly people. European journal of clinical nutrition 67 (10): 1050-5, 2013. 
14. Sadek KM, Shaheen H: Biochemical efficacy of vitamin D in ameliorating endocrine and metabolic disorders in diabetic rats. Pharmaceutical biology 52 (5): 591-6, 2014.

15. Ti Y, Xie G-l, Wang Z-h, Bi X-l, Ding W-y, Wang J, et al:: TRB3 gene silencing alleviates diabetic cardiomyopathy in a type 2 diabetic rat model. Diabetes 60 (11): 2963-74, 2011.

16. Li H, Shen Z, Lu Y, Lin F, Wu Y, Jiang Z: Muscle NT-3 levels increased by exercise training contribute to the improvement in caudal nerve conduction velocity in diabetic rats. Molecular medicine reports 6 (1): 69-74, 2012.

17. Head SI, Arber M: An active learning mammalian skeletal muscle lab demonstrating contractile and kinetic properties of fast-and slowtwitch muscle. Advances in physiology education 37 (4): 405-14, 2013.

18. Chan S, Head SI: Age-and gender-related changes in contractile properties of non-atrophied EDL muscle. PLoS One 5 (8): e12345-55, 2010.

19. Barton ER, Lynch G, Khurana T, Raymackers J, Dorchies O, Carlson G: Measuring isometric force of isolated mouse muscles in vitro. Treat-NMD Neuromuscular Network 1 (002): 14, 2008.

20. Saleh S, El-Maraghy N, Reda E, Barakat W: Modulation of diabetes and dyslipidemia in diabetic insulin-resistant rats by mangiferin: role of adiponectin and TNF- $\alpha$. Anais da Academia Brasileira de Ciências 86 (4): $1935-$ $48,2014$.

21. Klionsky DJ, Abdelmohsen K, Abe A, Abedin MJ, Abeliovich H, Acevedo Arozena A, et al.: Guidelines for the use and interpretation of assays for monitoring autophagy. Autophagy 12 (1): 1-222, 2016.

22. Bancroft J, Gamble M: Hematoxylin and eosin stains. 6th (ed), New York, Churchill Livingstone, 2007.

23. Andersen H, Schmitz O, Nielsen S:

Decreased isometric muscle strength after acute hyperglycaemia in Type 1 diabetic patients. Diabetic medicine 22 (10): 1401-7, 2005.

\section{Xiang X, Wang Z, Zhu Y, Bian L, Yang}

Y: Dosage of streptozocin in inducing rat model of type 2 diabetes mellitus. Wei sheng yan jiu= Journal of hygiene research 39 (2): 138-42, 2010.

25. Mabhida SE, Johnson R, Ndlovu M, Louw J, Opoku A, Mosa RA: Molecular basis of the anti-hyperglycemic activity of RA-3 in hyperlipidemic and streptozotocin-induced type 2 diabetes in rats. Diabetology \& Metabolic Syndrome 11 (1): 27, 2019.

26. Hamilton B: Vitamin D and athletic performance: the potential role of muscle. Asian journal of sports medicine 2 (4): 211-19, 2011.

27. Mehta BK, Singh KK, Banerjee S: Effect of exercise on type 2 diabetes-associated cognitive impairment in rats. International Journal of Neuroscience 129 (3): 252-63, 2019.

28. Han TS, Sattar N, Lean M: Assessment of obesity and its clinical implications. Bmj 333 (7570): 695-8, 2006.

29. He Y, Liu Y, Wang Q-Z, Guo F, Huang

F, Ji L, et al.: Vitamin D3 Activates Phosphatidylinositol-3-Kinase/Protein Kinase B via Insulin-Like Growth Factor-1 to Improve Testicular Function in Diabetic Rats. Journal of diabetes research 2019: 8, 2019.

30. Borges ME, Ribeiro AM, Pauli JR, Arantes LM, Luciano E, de Moura LP, et al.: 
Cerebellar Insulin/IGF-1 signaling in diabetic rats: Effects of exercise training. Neuroscience letters 639: 157-61, 2017.

31. Bai P, Nagy L, Fodor T, Liaudet L, Pacher P: Poly (ADP-ribose) polymerases as modulators of mitochondrial activity. Trends in Endocrinology \& Metabolism 26 (2): 75-83, 2015.

32. Abdel-Mohsen MA, El-Braky AA-A, Ghazal AAE-R, Shamseya MM: Autophagy, apoptosis, vitamin $\mathrm{D}$, and vitamin $\mathrm{D}$ receptor in hepatocellular carcinoma associated with hepatitis C virus. Medicine 97 (12): e0172-9, 2018.

33. Wu S, Sun J: Vitamin D, vitamin D receptor, and macroautophagy in inflammation and infection. Discovery medicine 11 (59): 325-35, 2011.

\section{Ogata T, Oishi Y, Higuchi M, Muraoka}

I: Fasting-related autophagic response in slow-and fast-twitch skeletal muscle. Biochemical and biophysical research communications 394 (1): 136-40, 2010.

35. Virgen-Ortiz A, Apolinar-Iribe A, Muñiz J: Gender-effect on the contractile properties of skeletal muscle in streptozotocininduced diabetic rats. Journal of musculoskeletal \& neuronal interactions 18 (2): 255-61, 2018.

36. Huang $\mathbf{L}$, Chen $\mathbf{L}$, Qiu Y, Li $\mathbf{S}$ : Abnormalities in the fiber composition and contractility in diabetic skeletal muscles. Int J Clin Exp Med 11 (2): 753-63, 2018.

\section{Imam KA, Sarwar M, Wali U, Siddique}

L, Perveen S: Gender and contractile functions of slow and fast skeletal muscles in streptozotocin induced diabetic sprague dawley Rats. Romanian
Journal of Diabetes Nutrition and Metabolic Diseases 19 (4): 417-24, 2012.

38. Aleem SB, Hussain MM, Farooq Y: Levo-carnitine reduces oxidative stress and improves contractile functions of fast muscles in type 2 diabetic rats. Iranian biomedical journal 17 (1): 29-35, 2013.

39. Ashfaq M: Skeletal Muscle Fatigue in Slow and Fast Muscles of Type 2 Diabetic Sprague Dawley Rats. Journal of Rawalpindi Medical College 21 (4): 390-4, 2017.

40. Polly P, Tan TC: The role of vitamin D in skeletal and cardiac muscle function. Frontiers in physiology 5: 145-52, 2014.

41. Eshima H, Tamura Y, Kakehi S, Nakamura K, Kurebayashi N, Murayama T, et al.: Dysfunction of muscle contraction with impaired intracellular $\mathrm{Ca} 2+$ handling in skeletal muscle and the effect of exercise training in male $\mathrm{db} / \mathrm{db}$ mice. Journal of Applied Physiology 126 (1): 170-82, 2019.

42. Hany K: Effect of diabetes mellitus on the structure of skeletal muscle in adult male Albino rats and the protective role of chromium: histological and immunohistochemical study. Egyptian Journal of Histology 31 (2): 341-53, 2008.

43. Stratos I, Li Z, Herlyn P, Rotter R, Behrendt A-K, Mittlmeier T, et al.: Vitamin D increases cellular turnover and functionally restores the skeletal muscle after crush injury in rats. The American journal of pathology 182 (3): 895-904, 2013. 